\title{
Long non-coding RNA CCAT2 is associated with poor prognosis in hepatocellular carcinoma and promotes tumor metastasis by regulating Snail2- mediated epithelial-mesenchymal transition
}

\author{
This article was published in the following Dove Press journal: \\ OncoTargets and Therapy \\ 24 February 2017 \\ Number of times this article has been viewed
}

\section{Yongfu Xu* \\ Binfeng Wang* \\ Fabiao Zhang \\ Aidong Wang \\ Xuefeng Du \\ Peng $\mathrm{Hu}$ \\ Yu Zhu \\ Zheping Fang}

Department of Hepatobiliary Surgery, Taizhou Hospital of Zhejiang Province, Wenzhou Medical University, Linhai,

Zhejiang, People's Republic of China

*These authors contributed equally to this work
Correspondence: Zheping Fang Department of Hepatobiliary Surgery, Taizhou Hospital of Zhejiang Province, Wenzhou Medical University, No 150 Ximen Road, Linhai, Zhejiang 317000, People's Republic of China

Tel +8657685 I 20120

Fax +865768519 9876

Email fangzhepingtz@I63.com

\begin{abstract}
Increasing evidence has demonstrated that aberrant expressions of long non-coding RNAs (lncRNAs) are involved in various malignancies, including hepatocellular carcinoma (HCC). This study aimed to investigate the role of lncRNA colon cancer-associated transcript 2 (CCAT2) in the progression of HCC. Quantitative real-time polymerase chain reaction analysis confirmed that CCAT2 was upregulated in HCC cell lines and cancerous tissues compared with normal liver cell line and adjacent normal tissue samples. The level of CCAT2 was positively associated with tumor-node-metastasis stages and vessel invasion. Survival analyses revealed that high CCAT2 expression predicted poor prognostic outcomes, serving as an independent prognostic factor for HCC patients. Patients with high CCAT2 expression had a 1.849-fold increased risk of death compared with those with low CCAT2 expression. Moreover, we also found that knockdown of CCAT2 expression reduced cell migration and invasion in vitro. We further demonstrated that CCAT2 played a key role in enhancing the epithelial-mesenchymal transition (EMT) through the regulation of vimentin, E-cadherin and transcription factor snail2 expression. Taken together, our findings showed that high CCAT2 expression is associated with poor survival in HCC patients. CCAT2 promotes HCC progression by regulating Snail2-induced EMT. CCAT2 may be a prognostic biomarker and therapeutic target for HCC.
\end{abstract}

Keywords: long non-coding RNA, CCAT2, hepatocellular carcinoma, epithelial-mesenchymal transition, survival

\section{Introduction}

Hepatocellular carcinoma (HCC) has become the most common leading cause of cancer-associated mortality globally. ${ }^{1}$ Although in recent years there are mounting progresses in clinical treatment for $\mathrm{HCC}$, the 5-year survival rate of $\mathrm{HCC}$ patients is still poor and exceeds 600,000 people dying of HCC each year. ${ }^{2}$ Uncontrolled tumor metastasis, frequent intrahepatic propagation and extrahepatic progression are the main causes for the poor prognosis of HCC. Identification of the prognostic predictors and key molecular mechanisms that influence the metastasis and progression of HCC will be meaningful.

In the human genome, $\sim 2 \%$ of transcripts can be translated into proteins, whereas 98\% of transcripts are non-coding RNAs, including microRNAs (miRNAs) and long non-coding RNAs (lncRNAs). ${ }^{3}$ The lncRNAs are $>200$ nucleotides in length and unable to be translated into proteins. Increasing evidence has demonstrated that 
lncRNAs can function as oncogenes or tumor suppressors, ${ }^{4,5}$ which could regulate downstream genes at different levels, including chromatin modification and transcriptional and posttranscriptional processing. ${ }^{6-8}$ Colon cancer-associated transcript 2 (CCAT2), a novel lncRNA mapping to 8q24 genomic region, was first discovered by Ling et al. ${ }^{9}$ They showed that CCAT2 was highly overexpressed in microsatellite-stable colorectal cancer and promoted tumor growth, metastasis and chromosomal instability. Overexpression of CCAT2 can also promote the metastasis and invasion of breast, lung and stomach cancer cells and influence the prognosis of breast and stomach cancers. ${ }^{10-12}$ However, the biological function and clinical significance of CCAT2 in HCC remain unknown.

Therefore, we performed a study to investigate the role of CCAT2 in HCC and its potential prognostic value. We found that the expression of CCAT2 was upregulated in HCC cell lines and tissues, and high CCAT2 expression was associated with advanced tumor-node-metastasis (TNM) stage, frequent vessel invasion and poor patient prognosis. Decreased CCAT2 expression in HCC cells significantly suppressed cell migration and invasion. Furthermore, we also showed that alteration of CCAT2 expression can influence E-cadherin, vimentin and snail2 levels, indicating that CCAT2 affected HCC progression partly through snail2induced epithelial-mesenchymal transition (EMT).

\section{Materials and methods}

\section{Clinical specimens}

A total of 96 pairs of $\mathrm{HCC}$ and matched paracancerous tissues were consecutively collected from patients who received surgical resection of HCC from September 2004 to October 2010 at Taizhou Hospital of Zhejiang Province, Affiliated Hospital of Wenzhou Medical University. The clinical and pathological characteristics were obtained from patient charts. None of the patients had received preoperative radiotherapy, chemotherapy or any other medical intervention. Tumors were staged according to the Seventh Edition of the Cancer Staging Manual by the American Joint Committee on Cancer. Among the patients, 53.1\% (51/96) were diagnosed with TNM stage I, 37.5\% (36/96) with TNM stage II, 9.4\% (9/96) with TNM stage III and no case were in stage IV. The median follow-up for all patients was 39.5 months (range, 5.2-60 months), and 56 cancerassociated fatalities (58.3\%) were recorded. The study was approved by the Ethics Committee on Human Research of Taizhou Hospital of Zhejiang Province, and written informed consent was obtained from all the patients.
Quantitative real-time polymerase chain reaction ( $q R T-P C R)$

Total RNAs were extracted from paired HCC and noncancerous tissues or cultured cells using Trizol reagent (Invitrogen, Carlsbad, CA, USA) following the manufacturer's protocol. RT and qPCR kits (Takara, Dalian, China) were used to determine the expression of CCAT2 in tissue samples and cultured cells. qRT-PCR was done using SYBR1 Premix Ex Taq II (Tli RNaseH Plus) (Takara) on ABI 7900HT (Applied Biosystems, Foster City, CA, USA). The primers used in this study are shown in Table 1 . The relative expression of CCAT2 was normalized to reference glyceraldehyde-3phosphate dehydrogenase (GAPDH) and was calculated.

\section{Cell lines, small interfering RNA (siRNA) and transfection of HCC cells}

The HCC cell lines HepG2, SMMC772 and MHCC97H and the normal liver cell line MIHA were purchased from Fudan

Table I Primers used for qRT-PCR and siRNA oligonucleotides

\begin{tabular}{|c|c|}
\hline Genes & Sequences \\
\hline \multicolumn{2}{|c|}{ Primers used for qRT-PCR } \\
\hline \multicolumn{2}{|c|}{ ССAT2 } \\
\hline Sense & 5'-CCCTGGTCAAATTGCTTAACCT-3' \\
\hline Antisense & 5'-TTATTCGTCCCTCTGTTTTATGGAT-3' \\
\hline \multicolumn{2}{|l|}{ Vimentin } \\
\hline Sense & 5'-GAGAACTTTGCCGTTGAAGC-3' \\
\hline Antisense & 5'-GCTTCCTGTAGGTGGCAATC-3' \\
\hline \multicolumn{2}{|l|}{ E-cadherin } \\
\hline Sense & 5'-TACGCCTGGGGACTCCACCTA-3' \\
\hline Antisense & 5'-CCAGAAACGGAGGCCTGAT-3' \\
\hline \multicolumn{2}{|l|}{ Snail2 } \\
\hline Sense & 5'-TGTGCTTACCAACCAAATAGTATG-3' \\
\hline Antisense & 5'-GGCTCTGGGCGGGTACAAAG-3' \\
\hline \multicolumn{2}{|l|}{ GADPH } \\
\hline Sense & 5'-TGGTATCGTGGAAGGACTCAT-3' \\
\hline Antisense & 5'-GTGGGTGTCGCTGTTGAAGTC-3' \\
\hline \multicolumn{2}{|c|}{ sIRNA-CCAT2 } \\
\hline \multicolumn{2}{|c|}{ siRNAI } \\
\hline Sense & 5'-GCUAAGAGGAAACCACCUUTT-3' \\
\hline Antisense & 5'-AAGGUGGUUUCCUCUUAGCTT-3' \\
\hline \multicolumn{2}{|l|}{ siRNA2 } \\
\hline Sense & 5'-GCUCCACCUCUGACCAAUUTT-3' \\
\hline Antisense & 5'-AAUUGGUCAGAGGUGGAGCTT-3' \\
\hline \multicolumn{2}{|l|}{ siRNA3 } \\
\hline Sense & 5'-CCAGGCAAUAACUGUGCAATT-3' \\
\hline Antisense & 5'-UUGCACAGUUAUUGCCUGGTT-3' \\
\hline \multicolumn{2}{|c|}{ Negative control } \\
\hline Sense & 5'-UUCUCCGAACGUGUCACGUTT-3' \\
\hline Antisense & 5'-ACGUGACACGUUCGGAGAATT-3' \\
\hline
\end{tabular}

Abbreviations: qRT-PCR, quantitative real-time polymerase chain reaction; siRNA, small interfering RNA; CCAT2, colon cancer-associated transcript 2; GAPDH, glyceraldehyde-3-phosphate dehydrogenase. 
University Cancer Institute (Shanghai, China). The HCC cell lines were cultured in Dulbecco's Modified Eagle's Medium (DMEM) containing $10 \%$ fetal bovine serum (FBS) at $37^{\circ} \mathrm{C}$ in an atmosphere containing 5\% $\mathrm{CO}_{2}$. HepG2 and $\mathrm{MHCC} 97 \mathrm{H}$ cells were cultured in six-well plates and transfected with siRNA or negative control using Lipofectamine 2000 (Invitrogen) according to the manufacturer's instructions. The siRNA specifically targeting CCAT2 (siRNA-CCAT2) was synthesized (Invitrogen). The siRNA sequences are shown in Table 1.

\section{Monolayer wound-healing assay}

Cell migration was analyzed using a wound-healing assay. Treated HepG2 and MHCC97H cells were plated in six-well plates containing 10\% FBS medium. Once the cells reached 90\% density, the monolayer was scratched and then cultured in fresh medium for $48 \mathrm{~h}$. Mitomycin C was applied to inhibit the cell division. The migration distances of the cells were measured from the images (five fields) that were obtained at the indicated time point.

\section{Transwell invasion assay}

The invasion ability of HCC cell was performed using 24-well Transwells ( $8 \mathrm{~mm}$ pore size; Corning Life Sciences) coated with $1 \mathrm{mg} / \mathrm{mL}$ Matrigel (BD Sciences). The cells were seeded in the upper chamber of the wells in $100 \mu \mathrm{L}$ FBSfree medium, and $500 \mu \mathrm{L}$ of $20 \%$ FBS medium was added to the lower chambers. Following incubation for $24 \mathrm{~h}$, the cells remaining on the upper membrane were removed with cotton wool. Cells that had invaded through the membrane were stained with methanol and $0.1 \%$ crystal violet and photographed with a phase-contrast inverted microscope (Olympus, Tokyo, Japan). The cells from at least five random microscopic fields $(\times 40)$ were counted.

\section{Statistical analysis}

SPSS 20.0 software (IBM, Armonk, NY, USA) was used for statistical analysis. The significance of differences between groups was estimated by Student's $t$-test and $\chi^{2}$ test as appropriate. The survival curves were calculated by the Kaplan-Meier method with the log-rank test applied for comparison. Survival data were evaluated using univariate and multivariate Cox proportional hazards model. Variables with a value of $P<0.05$ in univariate analysis were used in subsequent multivariate analysis on the basis of Cox regression analyses. Pearson's correlation analyses were used to investigate the correlation among CCAT2 with snail2, vimentin and E-cadherin. Two-sided $P$-values were calculated, and a $P$-value $<0.05$ was considered statistically significant.

\section{Results \\ CCAT2 expression is upregulated in human HCC cell lines and tissues and correlated with prognosis of $\mathrm{HCC}$ patients}

As shown in Figure 1A, CCAT2 level in three cancer cell lines (HepG2, SMMC772 and MHCC97H) was significantly higher than that of normal liver cell line (MIHA). Among all 96 pairs of HCC patients, CCAT2 expression level was significantly upregulated in $64 \%(61 / 96)$ of tumors compared with adjacent normal tissues $(P<0.01)$ (Figure 1B).

To assess the correlation of CCAT2 expression with clinicopathological characteristics, all cases were divided into low $(n=48)$ or high $(n=48)$ CCAT2 expression groups based on the median of CCAT2 expression level. As shown in Table 2, the high CCAT2 expression group showed higher TNM stage $(P=0.041)$ and more frequent vessel invasion $(P=0.026)$ than the low CCAT2 expression group. Besides, CCAT2 was significantly overexpressed in patients with alcoholism history $(P=0.041)$. However, there was no significant correlation between CCAT2 expression and other clinicopathological parameters such as sex, age, tumor size and smoking $(P>0.05)$.

Figure $1 \mathrm{C}$ is a Kaplan-Meier survival analysis depicting the relationship between CCAT2 expression and the prognosis of HCC. The mean survival time for low CCAT2 expression groups was $47.433 \pm 2.188$ months, whereas that for high CCAT2 expression groups was only $31.267 \pm 3.196$ months $(P<0.01$ by log-rank test $)$.

Furthermore, a Cox proportional hazards model analysis was used to assess the prognostic parameters in patients with HCC. Multivariate analysis showed that, after TNM stage, vessel invasion, sex, age, tumor size and alcoholism were adjusted, CCAT2 expression was independently associated with OS, with a hazard ratio (HR) of 1.849 (95\% confidence interval [95\% CI], 1.064-3.213; $P=0.029$; Table 3).

\section{CCAT2 promotes HCC cell migration and invasion in vitro}

Migration and invasion ability strongly correlate with cancer progression and metastasis. To investigate whether CCAT2 had a functional role in facilitating HCC cell metastasis, we performed wound-healing assay and Transwell assay. CCAT2 expression was downregulated by siRNAmediated gene silencing in both HepG2 and MHCC97H cells. The knockdown of CCAT2 expression in the cells 
A

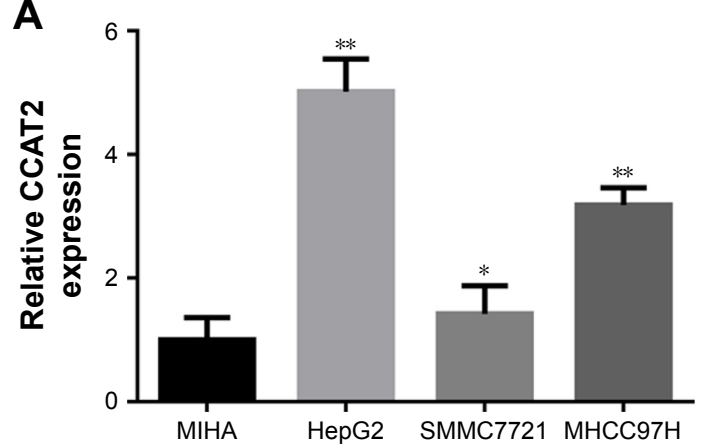

B

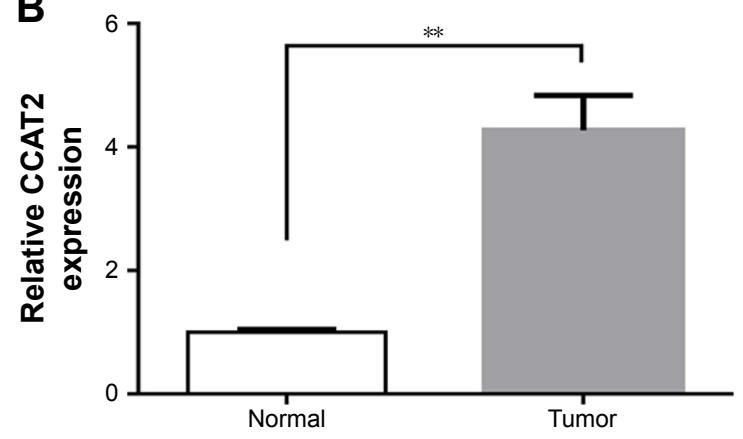

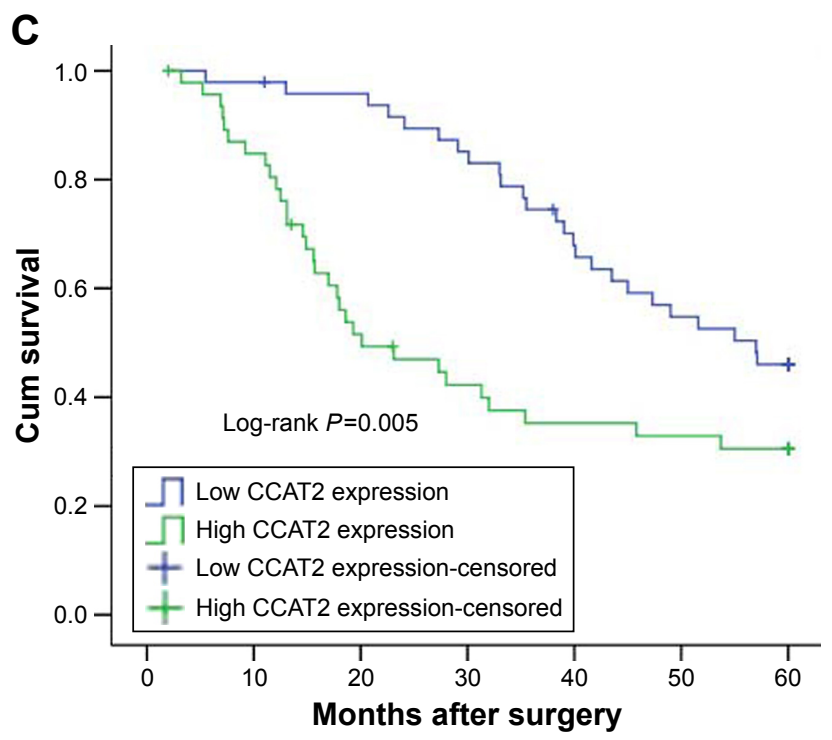

Figure I Increased expression of CCAT2 in HCC tissues and cell lines and its prognostic significance.

Notes: (A) qRT-PCR analysis of CCAT2 expression levels in human normal liver cell line (MIHA) and HCC cell lines (HepG2, SMMC772I and MHCC97H). GAPDH expression was assessed as an internal control. (B) The expression of CCAT2 was detected in 96 paired human HCC and adjacent normal tissues via qRT-PCR. The level of CCAT2 in tumor tissues was significantly higher than that in normal tissues. (C) Kaplan-Meier analysis of overall survival based on CCAT2 expression in all HCC patients. Patients with high levels of CCAT2 expression showed reduced survival times compared with patients with low expression ( $P=0.005$, log-rank test). $* P<0.05$, $* * P<0.01$. Abbreviations: CCAT2, colon cancer-associated transcript 2; HCC, hepatocellular carcinoma; qRT-PCR, quantitative real-time polymerase chain reaction; GAPDH, glyceraldehyde-3-phosphate dehydrogenase; Cum survival, cumulative survival.

was demonstrated by qRT-PCR (Figure 2A). As shown in Figure 2, low CCAT2 expression in HepG2 and MHCC97H cells, which were transfected with siRNA-CCAT2, exhibited a significantly lower scratch closure rate than that in control cells (Figure 2B). Moreover, compared with the control cells, low CCAT2 expression in HCC cells also showed markedly repressed invasion ability (Figure $2 \mathrm{C}$ ). These findings indicate that CCAT2 expression may play a pivotal role in the migration and invasion of HCC cells.

\section{CCAT2 promotes HCC cell EMT}

EMT plays crucial roles during cancer initiation and progression, especially in increasing invasion and migration ability of cancer cells. ${ }^{13,14}$ To further investigate the possible mechanism by which CCAT2 promotes HCC progression, the expression levels of EMT-related markers such as vimentin, E-cadherin and snail2 were detected by qRT-PCR in HCC tissues. As expected, the Pearson's correlation analysis showed that the expression of CCAT2 in HCC lesions was positively correlated with vimentin $(r=0.587, P<0.001)$ and snail2 $(r=0.494, P<0.001)$ and negatively related to E-cadherin $(r=-0.233, P=0.022)$. Next, we transfected CCAT2 siRNA into HepG2 cells and analyzed its effects on the expression of vimentin, snail2 and E-cadherin. We found that the expression levels of vimentin and snail 2 were significantly reduced in cells treated with siRNA-CCAT2. However, the expression of E-cadherin was increased after inhibition of CCAT2 (Figure 3). These data support our hypothesis that CCAT2 influences HCC progression by regulating snail2induced EMT.

\section{Discussion}

Increasing evidence links dysregulation of lncRNAs to diverse human diseases, including HCC. ${ }^{15-18}$ In this study, we found that CCAT2 was upregulated in HCC tissues and cell lines, compared with adjacent normal tissues and normal liver 
Table 2 Association of CCAT2 expression with clinicopathological characteristics of $\mathrm{HCC}$

\begin{tabular}{|c|c|c|c|}
\hline \multirow{2}{*}{$\begin{array}{l}\text { Clinical } \\
\text { parameters }\end{array}$} & \multicolumn{2}{|c|}{ CCAT2 expression } & \multirow{2}{*}{$\begin{array}{l}\text { Chi-squared test } \\
P \text {-value }\end{array}$} \\
\hline & Low* & High* & \\
\hline Age (years) & & & 0.102 \\
\hline$\leq 54$ & 29 & 21 & \\
\hline$>54$ & 19 & 27 & \\
\hline Sex & & & 0.820 \\
\hline Male & 34 & 35 & \\
\hline Female & 14 & 13 & \\
\hline Alcoholism & & & $0.04 I$ \\
\hline Negative & 30 & 20 & \\
\hline Positive & 18 & 28 & \\
\hline Smoking & & & 0.682 \\
\hline Negative & 27 & 25 & \\
\hline Positive & 21 & 23 & \\
\hline TNM stages & & & $0.04 I$ \\
\hline 1 & 31 & 21 & \\
\hline$\| / I I I$ & 17 & 27 & \\
\hline Vessel invasion & & & 0.026 \\
\hline Absence & 6 & 15 & \\
\hline Presence & 42 & 33 & \\
\hline Tumor size $(\mathrm{cm})$ & & & 0.214 \\
\hline$\leq 5$ & 23 & 17 & \\
\hline$>5$ & 25 & 31 & \\
\hline
\end{tabular}

Note: *Patients were divided into high (above the median) and low (below the median) CCAT2 expression groups according to the median expression level (I.89) of CCAT2.

Abbreviations: CCAT2, colon cancer-associated transcript 2; HCC, hepatocellular carcinoma; TNM, tumor-node-metastasis.

cell line. High CCAT2 expression was significantly correlated with aggressive tumor characteristics (higher tumor stage and vessel invasion) and poor prognosis. Moreover, multivariate analyses showed that CCAT2 expression was an independent prognostic factor for HCC patients. These findings support the concept that the CCAT2 expression level could serve as a prognostic biomarker for HCC.

CCAT2 expression is exhibited in different manners in various types of tumors. Qiu et al ${ }^{11}$ reported that $C C A T 2$ was a lung adenocarcinoma-specific lncRNA and not correlated with sex, smoking, TNM stage, tumor size or lymph node metastasis. However, Wang et al ${ }^{12}$ found that high CCAT2 expression was associated with higher incidence of lymph node metastasis and distance metastasis and predicted poor prognosis in gastric cancer. In addition, Redis et a ${ }^{10}$ revealed that CCAT 2 overexpression decreases the sensitivity of breast cancer cells to 5-fluorouracil (5-FU). Interestingly, CCAT2 expression in breast cancer was inversely correlated with nodal status, having the highest expression in lymph nodenegative disease. Therefore, CCAT2 may exert cancer-typedependent effects. Further studies are definitely required to examine the role of CCAT2 in different human tumors.

Recent studies indicate that IncRNAs play an important role in tumor metastasis. Knockdown of lncRNA SPRY4IT1 inhibited the migration and invasion of colorectal cancer cells and induced cell cycle arrest. ${ }^{19}$ The lncRNA FTX could inhibit HCC cell growth and metastasis both in vitro and in vivo. ${ }^{20}$ CCAT2 has also been shown to promote proliferation and invasion in breast, ${ }^{10}$ lung $^{11}$ and cervical cancer cells. ${ }^{21}$ We then determined the effect of CCAT2 in HCC cells. We found that downregulated expression of CCAT2 inhibited cell migration and invasion, in line with the findings of Zhou et al. ${ }^{22}$ However, compared with the study performed by Zhou et al, our study has two strengths: first, our study revealed that the expression of CCAT2 in HCC tissue is an independent prognostic factor for HCC; second, we found that, besides regulating migration, CCAT2 was involved in the pathogenesis of HCC by regulating EMT.

There are various mechanisms of CCAT2 being involved in the pathogenesis of cancer. Previous data showed that upregulation of CCAT2 might promote the proliferation and metastasis of cancer cells through enhancing the WNT pathway. ${ }^{9,23}$ CCAT2 could physically interact with TCF7L2, by which the expressions of miR-17-5p, miR-20a and MYC are regulated in colon cancer. ${ }^{9}$ To date, the detailed molecular mechanism through which CCAT2 contributes to HCC progression is still unclear. Recent studies have revealed that some lncRNAs can regulate tumor cell metastasis by affecting the EMT process in HCC. . $^{20,24,25}$ Therefore, we further analyzed the relationship between CCAT2 and EMT in HCC. Hallmarks

Table 3 Univariate and multivariate Cox regression analyses of CCAT2 for overall survival of patients with HCC ( $n=96$ )

\begin{tabular}{|c|c|c|c|c|}
\hline \multirow[t]{2}{*}{ Variables } & \multicolumn{2}{|l|}{ Univariate analysis } & \multicolumn{2}{|c|}{ Multivariate analysis } \\
\hline & HR (95\% CI) & $P$-value & HR (95\% Cl) & $P$-value \\
\hline Age (years) ( $\leq 54$ vs $>54)$ & $1.152(0.682-1.946)$ & 0.598 & & \\
\hline Sex (male vs female) & $0.752(0.4 \mid 0-1.380)$ & 0.358 & & \\
\hline Smoking (positive vs negative) & $0.581(0.333-1.012)$ & 0.055 & & \\
\hline Alcoholism (positive vs negative) & $1.518(0.898-2.567)$ & 0.119 & & \\
\hline Tumor size $(\leq 5 />5)$ & $1.548(0.900-2.661)$ & 0.114 & & \\
\hline TNM stages (I vs II/III) & $2.333(1.37 \mathrm{I}-3.969)$ & 0.002 & $1.992(1.045-3.795)$ & 0.036 \\
\hline Vessel invasion (absence vs presence) & $0.45 I(0.252-0.809)$ & 0.008 & $0.880(0.427-1.815)$ & 0.730 \\
\hline CCAT2 expression (high vs low) & $2.118(1.245-3.603)$ & 0.006 & $1.849(1.064-3.213)$ & 0.029 \\
\hline
\end{tabular}

Abbreviations: CCAT2, colon cancer-associated transcript 2; HCC, hepatocellular carcinoma; HR, hazard ratio; $\mathrm{Cl}$, confidence interval; TNM, tumor-node-metastasis. 
of EMT are the aberrant expression of E-cadherin, vimentin and the transcription factor, snail. We found that CCAT2 expression was positively correlated with vimentin and snail2 and negatively related to E-cadherin in HCC tissues, basically supporting our hypothesis that CCAT2 was involved in the pathogenesis of HCC via regulating EMT. Then, we examined the expression levels of snail2, E-cadherin and vimentin following inhibition of CCAT2 in HCC cells. We found that depletion of CCAT2 inhibited snail 2 and vimentin expression and promoted E-cadherin expression, indicating that CCAT2 mediated promoting effects on HCC cell metastasis possibly by affecting the Snail2-induced EMT. EMT is implicated in the promotion of tumor cell invasion and metastasis. Therefore, as an important regulator of EMT, CCAT2 could be a suitable candidate for intervention in the treatment of cancer, serving as a drug target. Drugs that could regulate the expression of CCAT2 have clinical application prospects.

\section{Conclusion}

We found that CCAT2 was upregulated in HCC tissues and cells and served as a negative prognostic factor in HCC patients. More importantly, we primarily confirmed
A

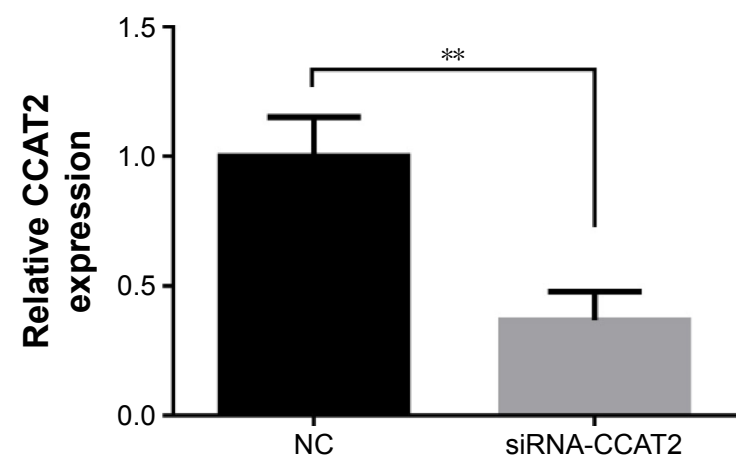

B
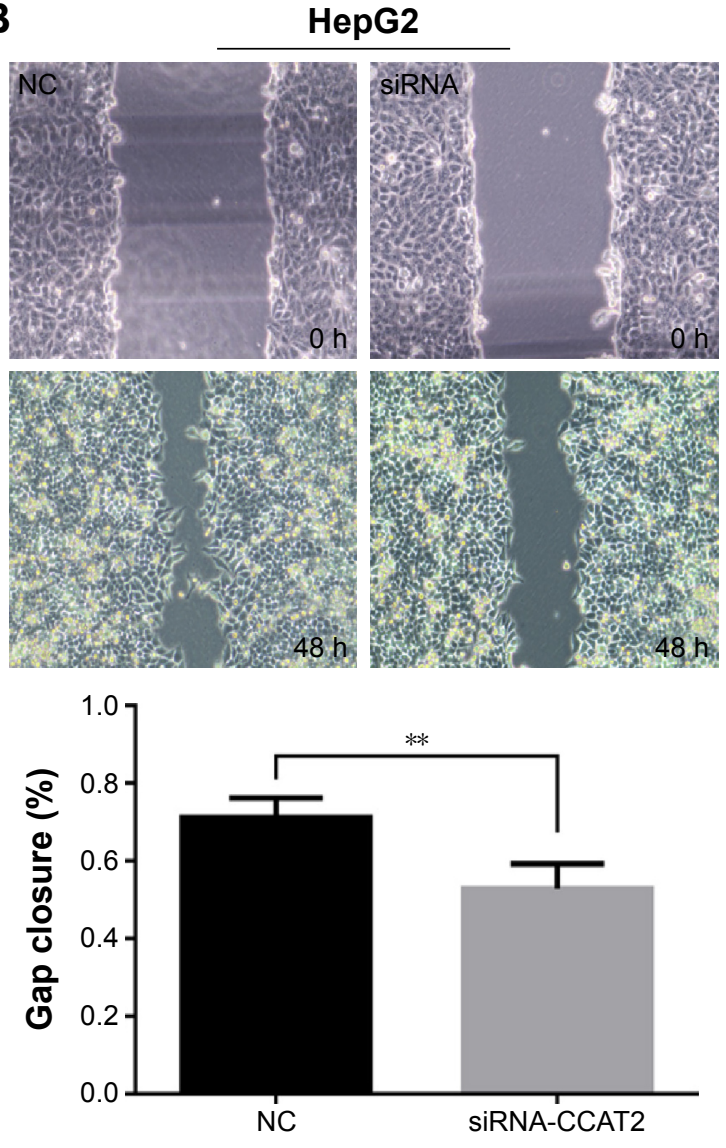

MHCC97H

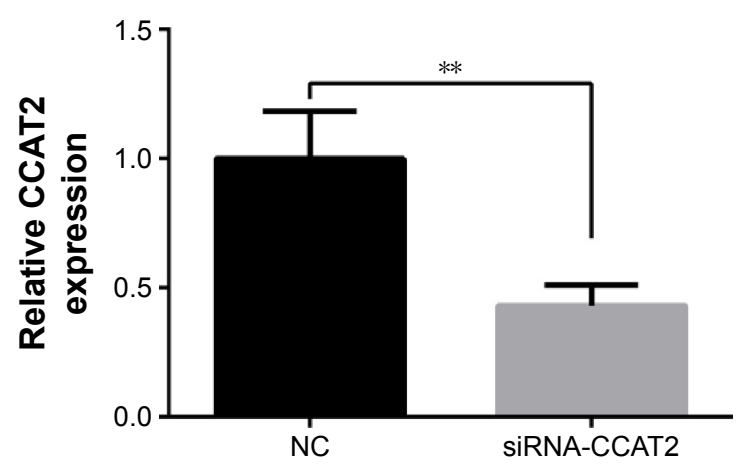

MHCC97H
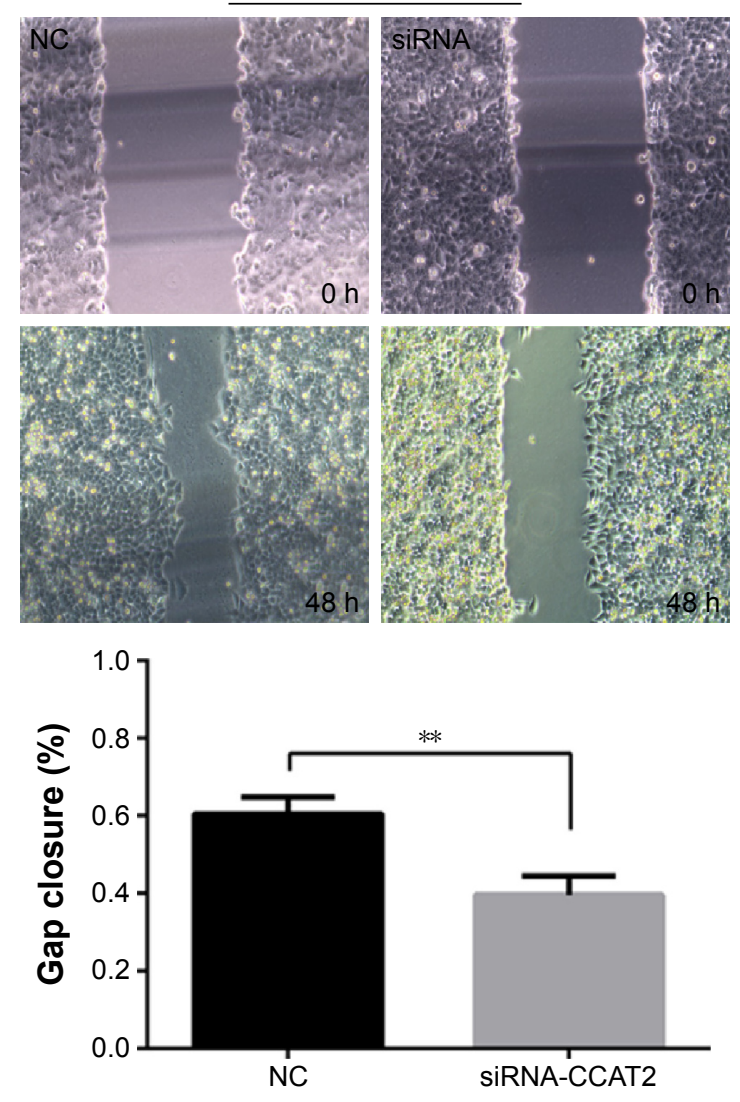

Figure 2 (Continued) 
C
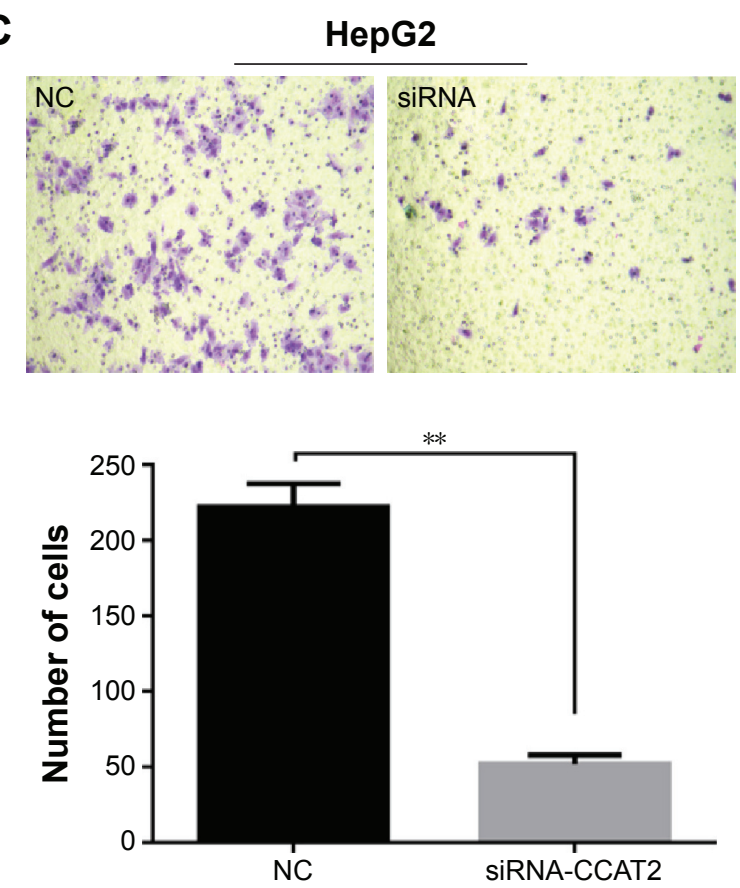

MHCC97H
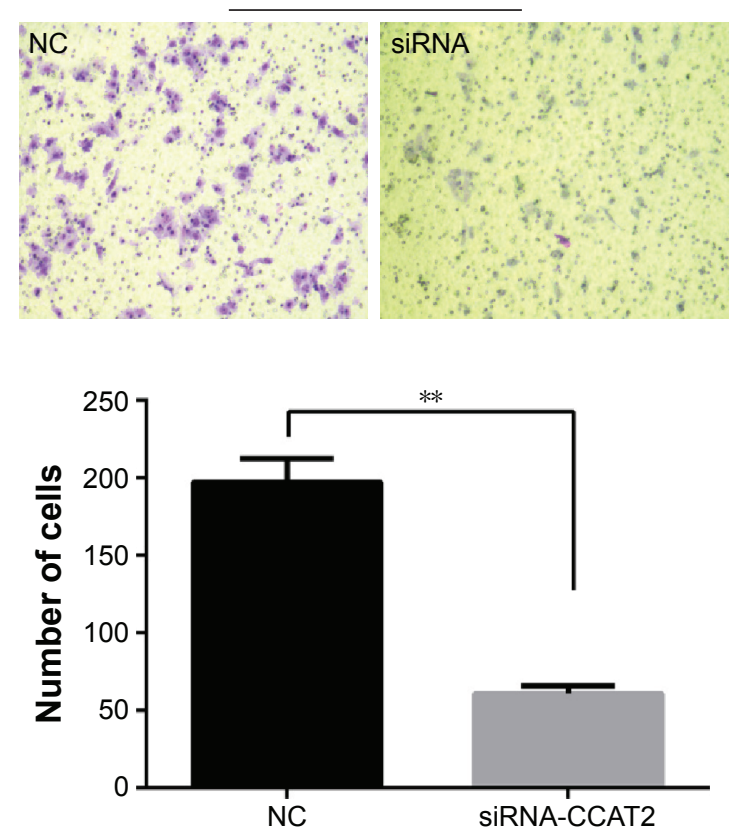

Figure 2 Effect of CCAT2 on HCC cells migration and invasion.

Notes: (A) The expression of CCAT2 in HepG2 and MHCC97H cells transfected with siRNA against CCAT2 was detected by qRT-PCR. (B and C) Wound-healing assay and Transwell assay indicated that siRNA-mediated silence of CCAT2 significantly inhibited cell migration and invasion, compared with negative control. Original magnification $\times 40$. $* * P<0.01$.

Abbreviations: CCAT2, colon cancer-associated transcript 2; HCC, hepatocellular carcinoma; siRNA, small interfering RNA; qRT-PCR, quantitative real-time polymerase chain reaction; NC, negative control.
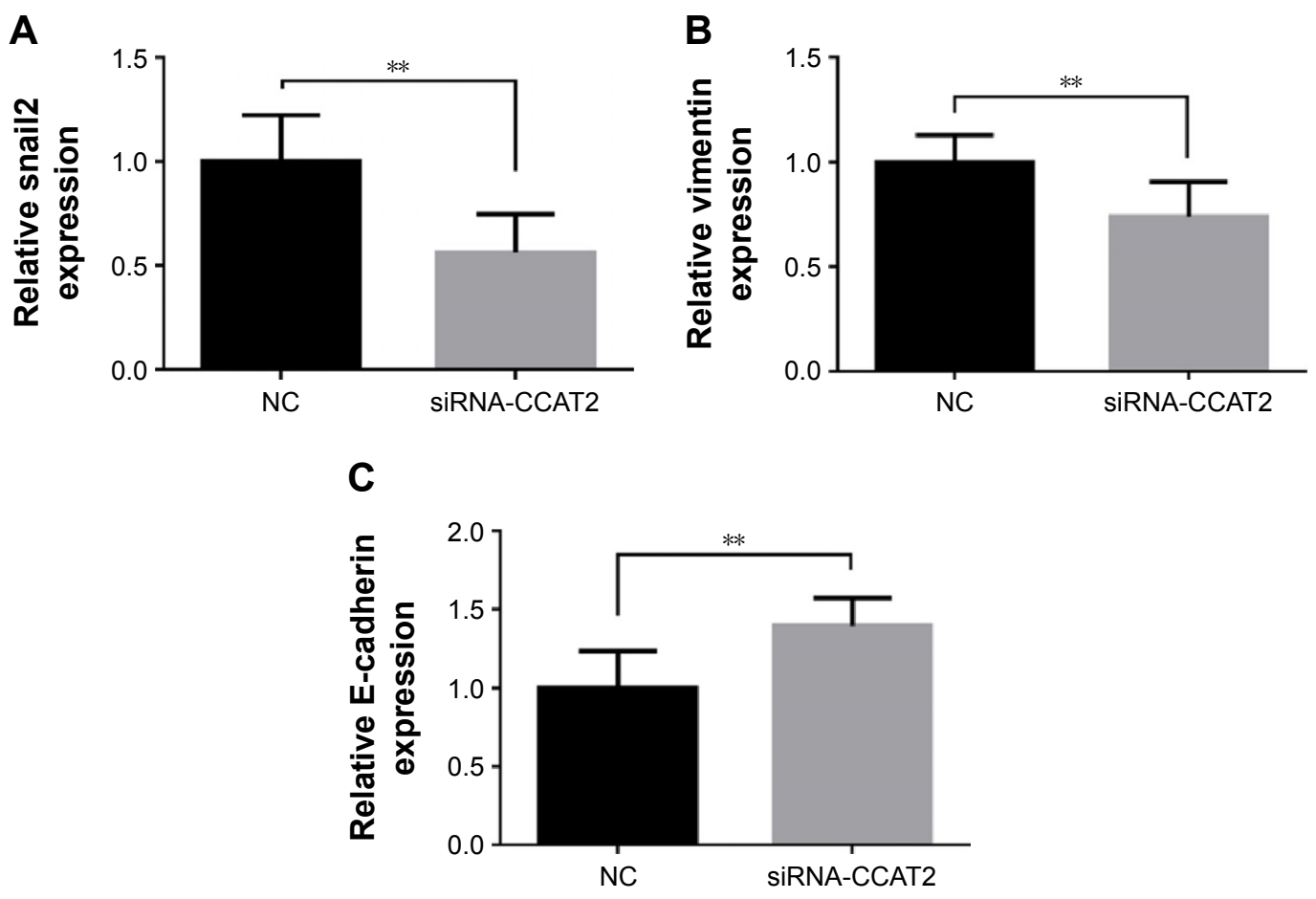

Figure 3 qRT-PCR analysis expression of snail2, vimentin and E-cadherin in HCC cells treated with siRNA-CCAT2.

Notes: The expression level of snail2 (A) and vimentin (B) was significantly reduced and that of E-cadherin (C) as upregulated in HCC cells after knockdown of CCAT2. $* * p<0.01$.

Abbreviations: qRT-PCR, quantitative real-time polymerase chain reaction; HCC, hepatocellular carcinoma; siRNA, small interfering RNA; CCAT2, colon cancer-associated transcript 2; NC, negative control. 
the regulatory mechanism of lncRNA CCAT2 in HCC that CCAT2 promoted tumor progression through increasing cancer cell migration and invasion ability in part by regulating Snail2-mediated EMT. Further insights into the functional and clinical implications of CCAT2 and its targets may propel the development of novel therapeutic strategies for HCC.

\section{Acknowledgments}

This project was supported by the Science Technology Program of Zhejiang Province on the Scientific Research Project (LY17H160069) and the Zhejiang Provincial Health Department Project (grant nos 2014KYA227 and 2017KY161).

\section{Author contributions}

Zheping Fang and Fabiao Zhang conceived and designed the study; Yongfu Xu and Binfeng Wang performed the majority of experiments and wrote the manuscript; Aidong Wang and Xuefeng Du contributed to the sample collection; Peng $\mathrm{Hu}$ and $\mathrm{Yu}$ Zhu performed the statistical analysis. All authors contributed toward date analysis, drafting and critically revising the paper and agree to be accountable for all aspects of the work.

\section{Disclosure}

The authors report no conflicts of interest in this work.

\section{References}

1. Forner A, Llovet JM, Bruix J. Hepatocellular carcinoma. Lancet. 2012;379(9822):1245-1255.

2. Minguez B, Lachenmayer A. Diagnostic and prognostic molecular markers in hepatocellular carcinoma. Dis Markers. 2011;31(3):181-190.

3. Cech TR, Steitz JA. The noncoding RNA revolution-trashing old rules to forge new ones. Cell. 2014;157(1):77-94.

4. Mitra SA, Mitra AP, Triche TJ. A central role for long non-coding RNA in cancer. Front Genet. 2012;3:17.

5. Wang J, Sun J, Wang J, et al. Long noncoding RNAs in gastric cancer: functions and clinical applications. Onco Targets Ther. 2016;9:681-697.

6. Mercer TR, Dinger ME, Mattick JS. Long non-coding RNAs: insights into functions. Nat Rev Genet. 2009;10(3):155-159.

7. Wilusz JE, Sunwoo H, Spector DL. Long noncoding RNAs: functional surprises from the RNA world. Genes Dev. 2009;23(13):1494-1504.

8. Han P, Chang CP. Long non-coding RNA and chromatin remodeling. RNA Biol. 2015;12(10):1094-1098.

9. Ling H, Spizzo R, Atlasi Y, et al. CCAT2, a novel noncoding RNA mapping to $8 \mathrm{q} 24$, underlies metastatic progression and chromosomal instability in colon cancer. Genome Res. 2013;23(9):1446-1461.

OncoTargets and Therapy

\section{Publish your work in this journal}

OncoTargets and Therapy is an international, peer-reviewed, open access journal focusing on the pathological basis of all cancers, potential targets for therapy and treatment protocols employed to improve the management of cancer patients. The journal also focuses on the impact of management programs and new therapeutic agents and protocols on
10. Redis RS, Sieuwerts AM, Look MP, et al. CCAT2, a novel long noncoding RNA in breast cancer: expression study and clinical correlations. Oncotarget. 2013;4(10):1748-1762.

11. Qiu M, Xu Y, Yang X, et al. CCAT2 is a lung adenocarcinoma-specific long non-coding RNA and promotes invasion of non-small cell lung cancer. Tumour Biol. 2014;35(6):5375-5380.

12. Wang CY, Hua L, Yao KH, Chen JT, Zhang JJ, Hu JH. Long non-coding RNA CCAT2 is up-regulated in gastric cancer and associated with poor prognosis. Int J Clin Exp Pathol. 2015;8(1):779-785.

13. Park MY, Kim KR, Park HS, et al. Expression of the serum response factor in hepatocellular carcinoma: implications for epithelial-mesenchymal transition. Int J Oncol. 2007;31(6):1309-1315.

14. Chen X, Lingala S, Khoobyari S, Nolta J, Zern MA, Wu J. Epithelial mesenchymal transition and hedgehog signaling activation are associated with chemoresistance and invasion of hepatoma subpopulations. J Hepatol. 2011;55(4):838-845.

15. Wang TH, Lin YS, Chen Y, et al. Long non-coding RNA AOC4P suppresses hepatocellular carcinoma metastasis by enhancing vimentin degradation and inhibiting epithelial-mesenchymal transition. Oncotarget. 2015;6(27):23342-23357.

16. Zhang JY, Weng MZ, Song FB, et al. Long noncoding RNA AFAP1AS1 indicates a poor prognosis of hepatocellular carcinoma and promotes cell proliferation and invasion via upregulation of the RhoA/ Rac2 signaling. Int J Oncol. 2016;48(4):1590-1598.

17. Zhu XT, Yuan JH, Zhu TT, Li YY, Cheng XY. Long noncoding RNA glypican 3 (GPC3) antisense transcript 1 promotes hepatocellular carcinoma progression via epigenetically activating GPC3. FEBS J. 2016; 283(20):3739-3754.

18. Zhou T, Gao Y. Increased expression of LncRNA BANCR and its prognostic significance in human hepatocellular carcinoma. World J Surg Oncol. 2016;14(1):8.

19. Cao D, Ding Q, Yu W, Gao M, Wang Y. Long noncoding RNA SPRY4-IT1 promotes malignant development of colorectal cancer by targeting epithelial-mesenchymal transition. Onco Targets Ther. 2016;9:5417-5425.

20. Liu F, Yuan JH, Huang JF, et al. Long noncoding RNA FTX inhibits hepatocellular carcinoma proliferation and metastasis by binding MCM2 and miR-374a. Oncogene. 2016;35(41):5422-5434.

21. Wu L, Jin L, Zhang W, Zhang L. Roles of long non-coding RNA CCAT2 in cervical cancer cell growth and apoptosis. Med Sci Monit. 2016;22:875-879.

22. Zhou N, Si Z, Li T, Chen G, Zhang Z, Qi H. Long non-coding RNA CCAT2 functions as an oncogene in hepatocellular carcinoma, regulating cellular proliferation, migration and apoptosis. Oncol Lett. 2016;12(1):132-138.

23. Cai Y, He J, Zhang D. Long noncoding RNA CCAT2 promotes breast tumor growth by regulating the Wnt signaling pathway. Onco Targets Ther. 2015;8:2657-2664.

24. Yuan JH, Yang F, Wang F, et al. A long noncoding RNA activated by TGF-beta promotes the invasion-metastasis cascade in hepatocellular carcinoma. Cancer Cell. 2014;25(5):666-681.

25. Wang TH, Yu CC, Lin YS, et al. Long noncoding RNA CPS1-IT1 suppresses the metastasis of hepatocellular carcinoma by regulating HIF-1alpha activity and inhibiting epithelial-mesenchymal transition. Oncotarget. 2016;7(28):43588-43603.

patient perspectives such as quality of life, adherence and satisfaction The manuscript management system is completely online and includes a very quick and fair peer-review system, which is all easy to use. Visit $\mathrm{http}: / /$ www.dovepress.com/testimonials.php to read real quotes from published authors. 